This item was submitted to Loughborough's Research Repository by the author.

Items in Figshare are protected by copyright, with all rights reserved, unless otherwise indicated.

\title{
Soft tissue motion influences skeletal loads during impacts
}

PLEASE CITE THE PUBLISHED VERSION

http://dx.doi.org/10.1097/JES.0b013e318168ead3

\section{PUBLISHER}

Lippincott Williams and Wilkins (@ The Amercian College of Sports Medicine)

\section{VERSION}

AM (Accepted Manuscript)

\section{LICENCE}

CC BY-NC-ND 4.0

\section{REPOSITORY RECORD}

Challis, John H., and Matthew T.G. Pain. 2019. "Soft Tissue Motion Influences Skeletal Loads During Impacts". figshare. https://hdl.handle.net/2134/8128. 
This item was submitted to Loughborough's Institutional Repository (https://dspace.lboro.ac.uk/) by the author and is made available under the following Creative Commons Licence conditions.

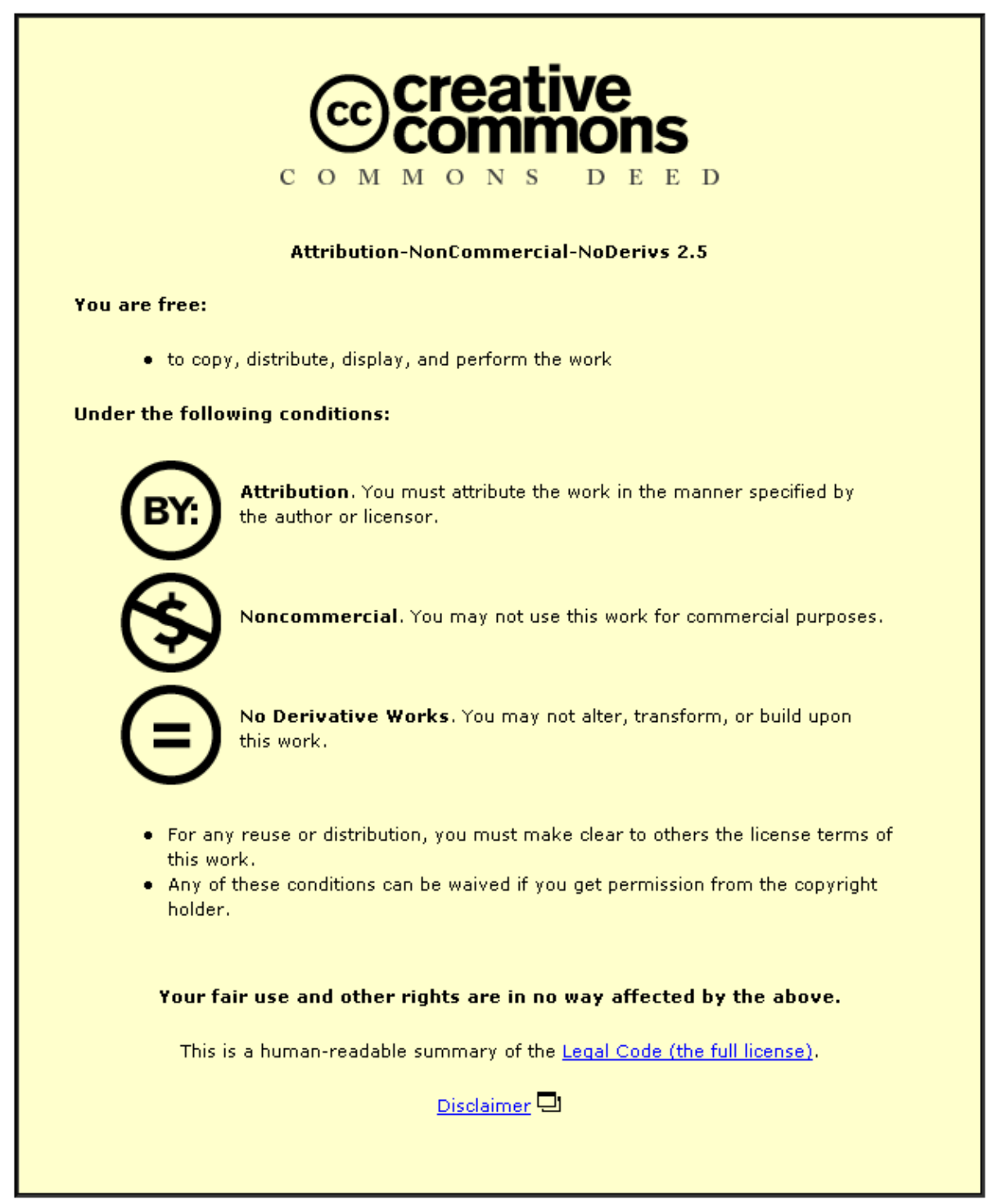

For the full text of this licence, please go to: http://creativecommons.org/licenses/by-nc-nd/2.5/ 


\title{
Soft tissue motion influences skeletal loads during impacts
}

\author{
John H. Challis ${ }^{1}$ and Matthew T.G. Pain ${ }^{2}$
}

${ }^{1}$ Biomechanics Laboratory, The Pennsylvania State University, University Park, PA 16802-3408, USA

${ }^{2}$ School of Sport \& Exercise Sciences, Loughborough University, Loughborough, LE11 3TU, U K

\begin{abstract}
Soft tissue motion occurs as impulsive loads are applied to the skeletal system. It has been demonstrated that the wave like motion of these wobbling masses can reduce the loads acting on the musculoskeletal system. This is an important concept to consider, whether the loads acting on the musculoskeletal system are being determined using either inverse or direct dynamics.
\end{abstract}

Keywords : soft tissue, wobbling mass, loads, musculoskeletal system

\section{Introduction}

As the foot strikes the ground during walking, running, and jumping, the foot experiences ground reaction forces. The ground reaction forces during the initial loading are typically large and applied rapidly (8), in animal studies such impulsive loading has been shown to produce joint degeneration (19). One marked feature of an impact is the motion of the soft tissue of the human body that it induces; this motion can be dramatic, for example during the impact of sumo wrestlers but it is also present during walking. Even as the muscles want to shorten they have wobble implying soft tissue motion in the opposite direction. The question therefore arises whether this soft tissue motion can confer any advantage to the musculoskeletal system.

There are many mechanisms via which the loading on the musculoskeletal system can be reduced including muscle activation and movement pattern modifications. The purpose of this review is to summarize the potential role that soft tissue motion can have in reducing musculoskeletal system loading. This soft tissue motion will be referred to as a wobbling mass.

\section{A mechanism for exploiting soft tissue}

Whenever two or more bodies contact with a relative velocity not equal to zero there is an impact. As the bodies come into contact intermolecular forces cause the bodies to decelerate. This process originates at the molecular level but rapidly propagates to being observable on the whole body level. If the unshod heel strike during running, walking or landing is examined, initially only the skin of the heel pad will undergo a forceful deformation but the movement of the skin will cause relative motion with respect to the underlying tissues and the impact process will be repeated at the molecular level to propagate the impact to adjacent tissue. The rate of this propagation is dependent on the stiffness of the bonds within a material, which in most solids is reflected in the overall stiffness of the material, and the stiffness of the connections between different materials. The forces are therefore transmitted through structures and tissues, and between structures and tissues, of the human body at different rates. These forces then cause changes in motion in accordance with Newton's laws to change the systems kinematics.

During impact with the ground the ground reaction force is determined by the sum of the products of the masses of each of the segments comprising a body and their accelerations. As 
the foot strikes the ground its downward velocity is rapidly arrested, causing acceleration of the whole skeletal system. If the system was rigid then accelerations would be uniform throughout the body. If the system is a collection of rigid bodies connected by joints then motion about joints can cause different accelerations throughout the system (20). But there is another factor which influences the ground reaction forces, the fact the segments are not rigid. As the foot strikes the downward velocity of the foot and shank are decreased but the soft tissue, particularly of the shank, may continue its motion downwards. Thus there are two acceleration components for the shank one high due to the skeleton the other low due to the soft tissue (figure 1). Each type of tissue can also exert a force directly on the other and this can result in the stiffer body applying a force to a region of the more compliant body before it is affected by forces transferred through itself. This can lead to shear forces from what would otherwise have been a compressive impact and lead to further complexity in the distribution of loads. The net effect is reduced acceleration of the overall system and reduced loading throughout the system.

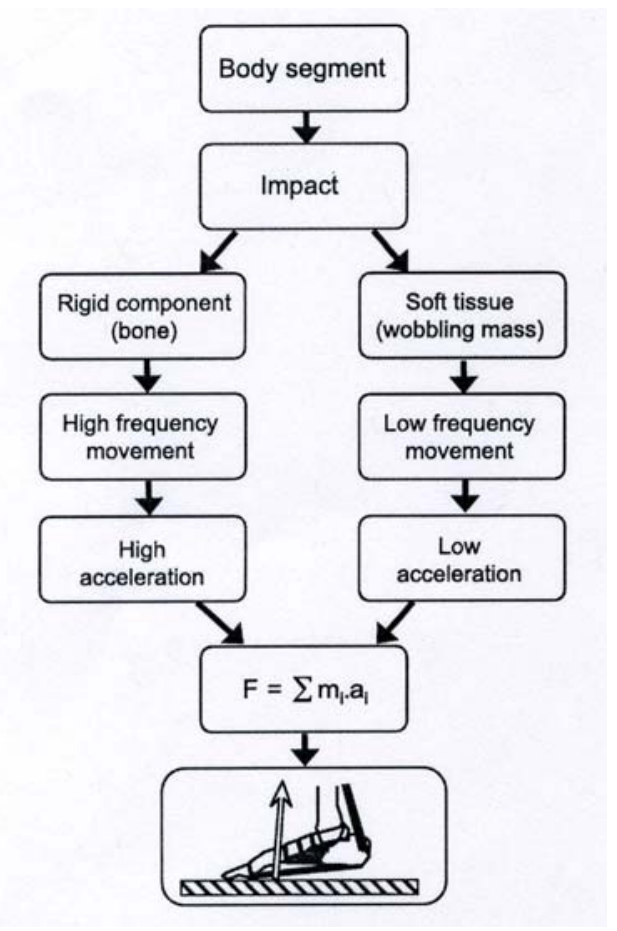

Fig. 1. A schematic representation how the wobbling masses can influence the ground reaction forces.

\section{Are the segments rigid?}

A human body segment can be considered to consist of skin, adipose tissue, muscle, connective tissues, and bone; of these components the bone can be considered fairly rigid but the other components, the soft tissues, are not. Clarys and Marfell-Jones (5) dissected the limbs of six cadavers, measured the masses of the individual segments and the components which comprise the segments. For all segments the skin was the smallest contributor to segment mass but for the thigh, shank, upperarm and forearm, bone was the next smallest contributor $(9.7 \%$ to 22.3\%).

Pain and Challis (15) measured the changes in the inertial properties of the shank as a consequence of the subject simply going from a relaxed muscle state to a tensed state. This 
action caused the center of mass of the shank to shift $1.7 \mathrm{~cm}$ proximally, with concomitant changes in segmental moments of inertia $(\approx 8 \%)$. A number of researchers have put inter-cortical pins into the bones of the lower limb and then compared the motion of markers placed on the skin relative to the motion of the bones. For example, Lafortune et al. (12) demonstrated up to 7 $\mathrm{cm}$ of soft tissue motion for the thigh. Such skin marker motion must be associated with the motion of the underlying soft tissues because skin has low stiffness compared with the stiffness of the muscle-tendon complexes (9).

The segments do have large non-rigid components which have their own motion during movement, but are the forces associated with these motions sufficiently large for us to be concerned about them? As a first approximation imagine this soft tissue motion relative to the bone has simple harmonic oscillation, the force $(F)$ associated with this soft tissue motion would be

$$
F=m(2 \pi f)^{2} d
$$

Where $m$ - mass of the soft tissue, $f$ - frequency of the motion, $d$ - displacement.

With a shank mass of $2.4 \mathrm{~kg}$, and a wobbling mass which comprises $65 \%$ of the mass of the shank (5), $m$ is equal to $1.56 \mathrm{~kg}$. During landings from a drop the frequency of the motion of the soft tissue can be high, for example a frequency of $14 \mathrm{~Hz}$ has been measured for shank soft tissue (13). Assuming a displacement of $0.017 \mathrm{~m}$ a force of $315 \mathrm{~N}$ is obtained. For the thigh with the greater soft tissue mass (5), greater soft tissue motion (12) and similar frequency content (18), the force associated with soft tissue motion would be much higher.

\section{What happens to the energy transferred to the soft tissue?}

An impact causes a transient deformation of the soft tissue, seen as a wave passing along the soft tissue of the limb. This waves occurs because the impact causes deformation at one end of the limb, and so an unbalanced force is produced, the visco-elastic properties of the soft tissue will attempt to return to equilibrium when the force is removed. An estimate of the energy carried by the tissue wave can be obtained by using the equation for the energy density of a nondispersive wave propagating in one direction,

$$
E d=\frac{1}{2} \rho \cdot A^{2} \cdot \omega^{2}
$$

Where $E d$ - energy density, $\rho$ - density of material the wave is propagating through,

$A$ - amplitude of wave and $\omega$ is angular frequency (radians/ s).

The relationship would not be this simple as the propagated waves are dispersive, a dispersive wave is one where the velocity of the wave is dependent on the frequency of the wave (10). The energy associated with a wave will eventually be passively dissipated within the medium, due to complex nature of the stress strain relation in the visco-elastic media comprising the soft tissue. Mechanical boundaries between tissues will also attenuate tissue wave motion due to mode conversions, reflections, and refractions not being one hundred percent efficient (10).

Pain and Challis (16) had a subject perform a karate strike onto a force plate. During the impact the intra-segmental motion of the forearm was quantified using a high speed motion analysis system and 28 surface mounted markers. The changes in area of the quadrilateral sectors defined by the markers was $11 \%$ and the deformations were approximately equal in the vertical and horizontal directions. The maximum intra-segmental linear marker motion was 1.7 
$\mathrm{cm}$. The intra-segmental motion had distinct frequency components around 14 and $20 \mathrm{~Hz}$. Soft tissue deformation could account for $70 \%$ of the energy lost from the forearm during these impacts, which in turn also accounted for the majority of the energy dissipation during the impact. An understanding of how much energy can be passively transferred or dissipated may prove useful in calculating potential injury to tissue. The skin marker artifact which compromises the kinematic analyses of human movement has to be examined in its own right for kinetic analyses as it is associated with forces not accounted for in rigid body analyses of human movement.

\section{Which wobbling mass model parameters are important?}

Models of soft tissue motion have conceptualized the human body as an underlying rigid skeletal system with other masses suspended from it by spring-dampers (11). A model was developed to examine which parameters in such models are important (17). The body segments in the model consisted of a rigid component representing the skeleton which had a second mass attached to it, representing the wobbling mass (figure 2). Some models have assumed that the wobbling mass motion does not influence the moments of inertia of the segment (7), but this is clearly not the case (15) as the motion of the wobbling masses does change all of the inertial properties of the modeled segments. Each wobbling mass was attached to the skeleton via two non-linear spring dampers, which represent the gross force interaction; this connection will be referred to as a "tendon", but does not strictly represent the mechanical properties of the tendons alone. Therefore the following parameters are part of a wobbling mass model: the inertial properties of the skeleton and wobbling masses, the properties of the tendon between the wobbling masses and the skeleton. Pain and Challis (17) performed a sensitivity analysis on these parameters to determine their influence on simulations of landing from a drop.
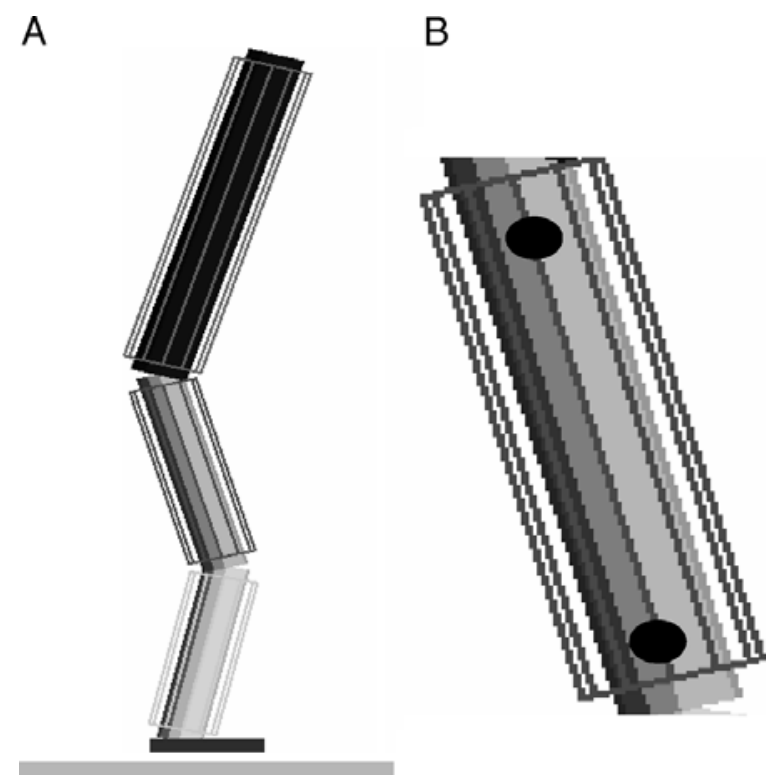

Fig. 2 a) The three link wobbling mass model. The inner solid segments represent the rigid skeleton. The outer line segments represent the wobbling mass material. b) An enlarged image of the thigh segment, it demonstrates the orientation of the skeleton and wobbling mass along with the location of the tendons (black dots). 
To examine the influence of the wobbling mass parameters a drop landing from $40 \mathrm{~cm}$ was simulated where the model landed on its heels. Such a landing is common in gymnastic activities and is not dissimilar to running gait where most landings are onto the heel (3). The body was modeled as three segments: one representing the head, arms and trunk segments, the second representing the upper leg, and the third representing the shank and foot. The rigid links were connected with revolute joints equipped with controllable joint actuators to emulate muscle actions. Each body segment consisted of a rigid component representing the skeleton which had a second mass attached to it, representing the wobbling mass (figure 2). The wobbling mass parameters were varied one at a time, and the change in the peak vertical ground reaction force (PVGRF) quantified. The perturbations were $\pm 20 \%$ of the initial parameter values as these were estimated to be greater than the error in these parameters.

Changing the ratio of soft tissue to bone mass had a large effect on the peak vertical ground reaction force, with a $20 \%$ increase in bone mass causing a $13 \%$ increase in force, and a $20 \%$ decrease in bone mass causing a 13\% decrease. This arises due to the initial impact peak being mainly due to deceleration of the rigid body skeleton from force applied to the foot-shank segment from the ground-heel pad interaction. The stiffness of the model tendons connecting the rigid to the wobbling masses did not have a large influence on the peak ground reaction force, with a $20 \%$ increase in stiffness resulting in a less than $1 \%$ change in the peak ground reaction force. Even when tendon stiffness was increased by an order of magnitude the peak vertical ground reaction force only increased by slightly more than $1 \%$.

\section{An example}

Using the model described in the previous section landings from a drop were simulated and compared to the performance of an experimental subject (18). The model parameters were determined independently of the model.

Table 1. The inertia parameters for the bone and soft tissue calculated for the simulation model

\begin{tabular}{|l|l|l|l|l|l|}
\hline Body Segment & Segment Type & $\begin{array}{l}\text { Mass (\% Body } \\
\text { Mass) }\end{array}$ & $\begin{array}{l}\text { Moment of } \\
\text { Inertia } \\
\text { (kgIms2) }\end{array}$ & $\begin{array}{l}\text { Segment } \\
\text { Length (m) }\end{array}$ & $\begin{array}{l}\text { Center of Mass } \\
\text { (\% Length) }\end{array}$ \\
\hline Foot & Whole & 2.5 & $3.42 \times 10^{-3}$ & 0.265 & 47.7 \\
\hline Shank & Bone & 3.1 & $3.80 \times 10^{-3}$ & 0.410 & 43.3 \\
\hline & Soft tissue & 6.3 & 0.0132 & 0.410 & 43.3 \\
\hline Thigh & Bone & 4.9 & 0.0570 & 0.425 & 43.3 \\
\hline Trunk & Soft tissue & 15.3 & 0.240 & 0.425 & 43.3 \\
\hline & Bone & 7.1 & 0.447 & 0.863 & 38.4 \\
\hline
\end{tabular}

The inertial properties of the segments were determined using regression equations presented in the literature $(4,22)$. The partitioning of segment mass to rigid and wobbling mass components was based on cadaver data $(5,6)$. The properties of the tendon between the wobbling and rigid model components masses were determined by comparing soft tissue motion of a subject and the model during stamping motions onto a force plate while soft tissue motion was tracked using arrays of retro-reflective markers placed on relevant segments. The heel pad 
model was adopted from Pain and Challis (15) which in turn was based on the cadaver data (1). The simulations were for a drop from $0.43 \mathrm{~m}$ onto a solid surface (force plate) for an $85 \mathrm{~kg}$ subject. The model's initial configuration and velocity at impact were determined from subject kinematics; the model parameters are presented in Table 1.

The peak vertical ground reaction for the subject was 16.4 body weights and 16.2 bodyweights for the model (figure 3). If the leg segments were made solid, so the model only had a wobbling mass at the trunk, the peak vertical ground reaction force increased to 31.4 bodyweights. If all wobbling masses were rigidly fixed to the rigid skeleton then the peak vertical ground reaction force increased to 40.5 bodyweights. The wobbling masses have a large influence on the loading on the system. In a similar fashion the resultant joint moments and forces were much larger for the rigid model than the wobbling mass model (table 2).

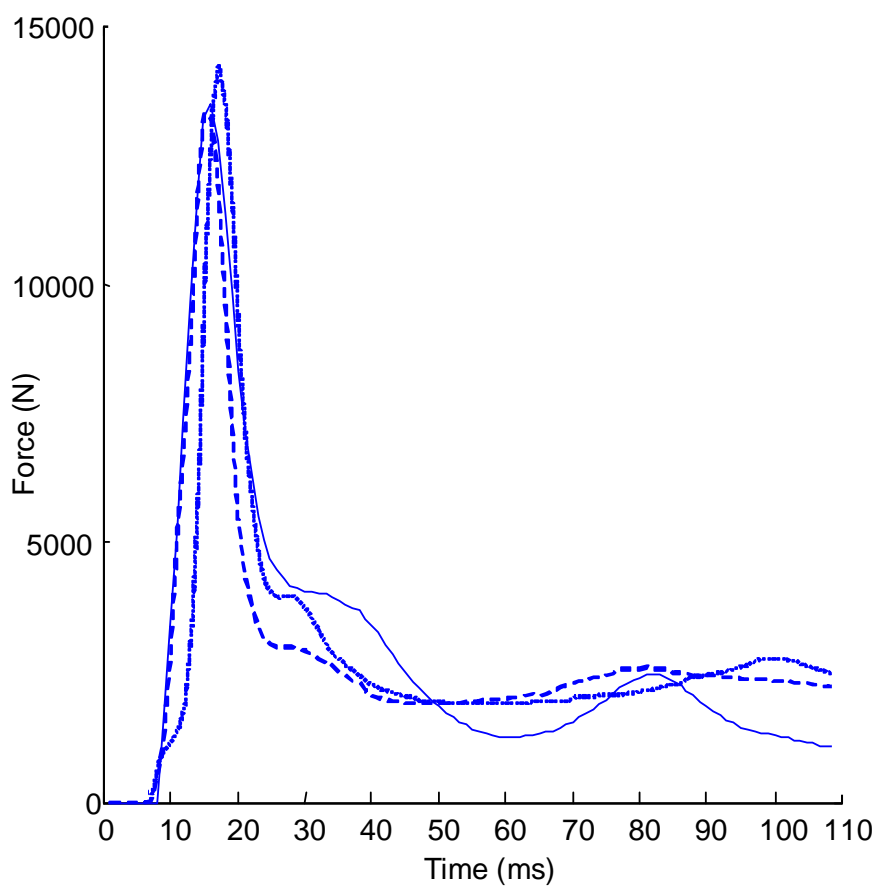

Fig. 3. The vertical ground reaction force curves for two trials by the experimental subject (dotted line and dashed line) and the wobbling mass model (solid line).

Table 2. The peak joint torques and forces for the wobbling mass and rigid body models

\begin{tabular}{|c|c|c|c|c|}
\hline \multirow{2}{*}{ Joint } & \multicolumn{2}{|c|}{ Joint Moment (N.m) } & \multicolumn{2}{c|}{ Force (N) } \\
\cline { 2 - 5 } & Wobbling & Rigid & Wobbling & Rigid \\
\hline Ankle & -228 & -370 & 11080 & 17140 \\
\hline Knee & 267 & 500 & 7720 & 7700 \\
\hline Hip & -240 & -460 & 5100 & \\
\hline
\end{tabular}


The wobbling masses of each of the segments reduces the effective mass of the segment contributing to the loads. The simulated landings had little joint motion and of course subjects can modify their muscular activations to soften the landing (21), or even modify the active acceleration of a segment into the impact or away from it. Even under these conditions the wobbling masses can still act to reduce system loading.

\section{Conclusion}

Soft tissue motion does occur and it has been demonstrated that under certain conditions this wobbling mass tissue motion helps reduce the loads acting on the musculoskeletal system. This is an important concept to consider, whether the loads acting on the musculoskeletal system are being determined using either inverse or direct dynamics. Researchers interested in gait analysis have examined ways of removing the influence of soft tissue motion on the positions of markers placed on the skin designed to track the motion of the underlying bones (2). Such analysis is appropriate when bone kinematics are of interest, but when this derived kinematics is used to determine kinetic quantities the segments are assumed rigid even though their lack of rigidity had to be accounted for in determining the kinematics. A set of forces are being ignored in such analyses which in many situations may be crucial to our understanding of the loads acting on the system and the movement pattern.

Significant challenges remain when examining human motion for activities where soft tissue motion is occurs. For those examining human motion experimentally, how can the influence of soft tissue motion be accounted for when examining musculoskeletal loads? For those using a simulation modeling approach, how can the appropriate model parameters be determined? As these questions are progressively addressed greater insight into the mechanics of human movement will be obtained.

\section{References}

1. Aerts, P., R. F. Ker, D. W. Ilsley, and R. M. Alexander. The mechanical properties of the human heel pad: A paradox resolved. Journal of Biomechanics. 28:1299-1308, 1995.

2. Alexander, E. J. and T. P. Andriacchi. Correcting for deformation in skin-based marker systems. Journal of Biomechanics. 34:355-361, 2001.

3. Cavanagh, P. R. and M. A. Lafortune. Ground reaction forces in distance running. Journal of Biomechanics. 13:397-406, 1980.

4. Challis, J. H. Accuracy of human limb moment of inertias and their influence on resultant joint moments. Journal of Applied Biomechanics. 12:517-530, 1996.

5. Clarys, J. P. and M. J. Marfell-Jones. Anthropometric prediction of component tissue mass in the minor limb segments of the human body. Human Biology. 58:761-769, 1986.

6. Clarys, J. P., A. D. Martin, and D. T. Drinkwater. Gross tissue weights in the human body by cadaver dissection. Human Biology. 56:459-473, 1984.

7. Cole, G. K., B. M. Nigg, A. J. van den Bogert, and K. G. M. Gerritsen. Lower extremity joint loading during impact in running. Clinical Biomechanics. 11:181-193, 1996.

8. Dickinson, J. A., S. D. Cook, and T. M. Leinhardt. The measurement of shock waves following heel strike while running. Journal of Biomechanics. 18:415-422, 1985.

9. Fung, Y. C. Biomechanics: Mechanical Properties of Living Tissue. New York: SpringerVerlag, 1981. 
10. Graff, K. F. Wave motion in elastic solids. Columbus: Ohio State University Press, 1975, xvii, 649.

11. Gruber, K., H. Ruder, J. Denoth, and K. Schneider. A comparative study of impact dynamics: wobbling mass model versus rigid body models. Journal of Biomechanics. 31:439444, 1998.

12. Lafortune, M. A., C. Lambert, and M. Lake. Skin marker displacement at the knee joint. In Second North American Congress on Biomechanics. Chicago, 1992.

13. Pain, M. T. G. and J. H. Challis. A high resolution technique for determining body segment inertial parameters and their variation due to soft tissue motion. Journal of Applied Biomechanics. 17:326-334, 2001.

14. Pain, M. T. G. and J. H. Challis. The influence of soft tissue movement on ground reaction forces, joint torques and joint reaction forces in drop landings. Journal of Biomechanics. 39:119-124, 2006.

15. Pain, M. T. G. and J. H. Challis. Measurement of the soft tissue motion of the thigh during an impact. In 3rd North American Congress on Biomechanics. University of Waterloo, 1998.

16. Pain, M. T. G. and J. H. Challis. The role of the heel pad and shank soft tissue during impacts: a further resolution of a paradox. Journal of Biomechanics. 34:327-333, 2001.

17. Pain, M. T. G. and J. H. Challis. Simulation of a wobbling mass model during an impact: a sensitivity analysis. Journal of Applied Biomechanics. 20:309-316, 2004.

18. Pain, M. T. G. and J. H. Challis. Soft tissue motion during impacts: Their potential contributions to energy dissipation. Journal of Applied Biomechanics. 18:231-242, 2002.

19. Radin, E. L., H. G. Parker, J. W. Pugh, R. S. Steinberg, I. L. Paul, and R. M. Rose. Response of joints to impact loading -- III : Relationship between trabecular microfractures and cartilage degeneration. Journal of Biomechanics. 6:51-54, 1973.

20. Wosk, J. and A. Voloshin. Wave attenuation in skeletons of young healthy persons. Journal of Biomechanics. 14:261-267, 1981.

21. Zatsiorsky, V. M. and B. I. Prilutski. Soft and stiff landing. In: Biomechanics $X-B$. B. Jonsson (Ed.) Illinois: Human Kinetics Publishers, Champaign, 1987, pp. 739-743.

22. Zatsiorsky, V. M., V. Seluyanov, and L. Chugunova. Methods of determining mass-inertial characteristics of human body segments. In: Contemporary Problems of Biomechanics. G. G. Chernyi and S. A. Regirer (Eds.) Moscow: Mir Publishers, 1990, pp. 272-291. 